\title{
Acute Pain Management in Patient on Intrathecal Opioid Infusion for Chronic Pain
}

\section{TO THE EDITOR:}

Intraspinal drug delivery (IDD) has been increasingly utilized since 1980s, initially in patients with cancer pain, and subsequently in patients with intractable, chronic, nonmaligmant pain. By infusing a small amount of opioid into the cerebrospinal fluid in close proximity to the receptor sites in the spinal cord, profound analgesia may be achieved while sparing some of the side effects due to systemic opioids. The introduction of intrathecal opioids has been considered one of the most important breakthroughs in pain management in the past 3 decades.

However, with the ever-increasing patient population nowadays, having implanted drug delivery pumps for their chronic pain, there appears to have a need for addressing acute pain management issues in this group of patients. The important questions to ask are: When patients, on intrathecal opioid infusion for chronic pain, go for surgical procedures, such as hip replacement or knee replacement, etc., what should be done with their intrathecal opioid regimen? Do we go up, come down, continue, or discontinue their routine intrathecal opioid infusion before surgery? What should we do for their postoperative pain? Can we still use routine modalities such as intravenous patientcontrolled analgesia (IV PCA) or epidural analgesia in such patients for their postoperative acute pain control? What about using intrathecal opioid for acute pain management since the patients already have intrathecal catheters implanted?

These are very pertinent and practical questions to raise, as we are encountering these situations more often than ever, because of the ever-increasing patient population with implanted intrathecal infusion pumps. There has been no literature, guidelines, consensus statements, expert opinions, or even recommendations published, to the best of my knowledge, pertaining to these clinical situations.

My own clinical approach has been keeping the intrathecal opioid infusion the same pre- and postsurgery, while utilizing IV opioid PCA for post opera- tive pain. This approach has also been utilized by some other interventional pain specialists who manage chronic pain patients with implanted pumps (J. Patrick Couch, MD, Rinoo V. Shah, MD, Srinivas Chiravuri, MD, personal communications). It seems reasonable to assume that the routine intrathecal infusion satisfies the opioid requirement for the chronic pain component, while the IV opioid PCA meets the additional opioid requirement for acute pain due to surgery. However, whether or not this assumption represents "standards of care" awaits further investigation.

Over the past 3 years, over 20 patients from my clinic, while on the same routine intrathecal opioid infusion therapy for chronic non-malignant pain, underwent surgical procedures such as lumbar fusion, cervical fusion, total hip replacement, total knee replacement, and received IV opioid PCA (morphine, hydromorphone) for post-operative pain, without encountering any complications or side effects of opioid overdose.

Nonetheless, it is still unclear what should be done in situations of above, even to interventional pain specialists who routinely manage patients with implanted pumps, not to mention those orthopedic surgeons who have no experience dealing with patients with implanted pain pumps for chronic pain, because of the lack of information or experience. There were quite a few times, in the past a few years, that I was consulted for pre- and post operative pain management because of surgeons feeling unsure what to do before and after operating on patients with implanted intrathecal opioid pumps.

ASIPP has been a true leader in establishing evidence-based practice guidelines in interventional pain management. In my opinion, the area of "Acute Pain Management in Patient on Intrathecal Opioid Infusion for Chronic Pain" is still "blank" and needs further investigation, due to the increasing popularity of intrathecal pain pumps used for varieties of chronic painful states. Hopefully, this correspondence will 
serve to draw the attentions of ASIPP experts to the increasingly encountered clinical scenario and call for an effort of collaboration from the experts and editors of ASIPP, possibly to bring about some guidelines, principles, or even expert opinions that can be used to direct clinical practice. This is especially important from medical-legal stand point, as complications/side effects do happen, even if good medicine is practiced. This underscores the importance of us interventional pain specialists to work out proper guidelines based on scientific knowledge under the leadership of ASIPP. Therefore, to better protect our patients and ourselves, I believe, it is time for this "blank" to be filled.

\author{
Xiulu Ruan, MD \\ Director \\ Clinical Research \& Electrodiagnostic Testing Services \\ Physicians' Pain Specialists of Alabama \\ Mobile, Alabama 36607 \\ Adjunct Assistant Professor of Neurology \\ College of Medicine \\ University of South Alabama \\ Mobile, Alabama 36688 \\ e-mail: xiuluruan@yahoo.com
}

\section{Ganglion Impar Blocks for Chronic Pelvic and Coccyx Pain}

\section{TO THE EDITOR:}

I applaud your journal and authors Toshniwal, Dureja, and Prashanth on the outstanding research article titled, "Transsacrococcygeal Approach to Ganglion Impar Block for Management of Chronic Perineal Pain: A Prospective Observational Study" (1). To advance the treatment of patients with coccydynia and other pelvic pain syndromes, this is exactly the type of research we need (well-designed, well-documented, thoroughly analysed and articulately written). The authors have very substantially added to the prior literature $(2,3)$ indicating that these ganglion impar injections can be safe and effective for treating coccydynia and other pelvic pain syndromes.

The authors' review of various approaches to performing ganglion impar blocks was very informative. I would add that there are 2 additional approaches to the ganglion impar, via either the first (2) or second (4) intracoccygeal joints, which I first published in 2006 and 2007, respectively. These may at times have advantages over approaching via the sacrococcygeal joint (SCJ). These intracoccygeal (or trans-coccygeal) approaches are evidence-based since Oh et al's anatomy article in 2004 shows that the ganglion Impar it is typically closer to the first intercoccygeal joint (ICJ-1, i.e. between the first and second coccygeal segments) (5) rather than at its previously believed location at the SCJ. In the hundreds of ganglion impar injections that I have performed, I typically note that the contrast tends to flow cephalad to the injection site, implying that a more inferior approach (e.g. via an ICJ) is best. Optimizing location would be particularly important when injecting neurolytic chemicals or performing radiofrequency ablation. Also, other research shows that ICJ-1 is much less likely to be fused than the SCJ is ( $12 \%$ of patients versus $51 \%$, respectively) (6) (probably accounting for the $19 \%$ of Toshniwal's patients who required an 18 gauge introducer needle at the SCJ). Lastly, in the lateral fluoroscopic view, the ICJ-1 is usually visualized more easily than the SCJ, since the bilateral sacral cornua and bilateral coccygeal cornua can obstruct the view (and obstruct the needle trajectory) at the SCJ.

I again congratulate the authors on their fantastic article. I hope that my comments may further facilitate pain physicians adopting these injections to help these patients find relief.

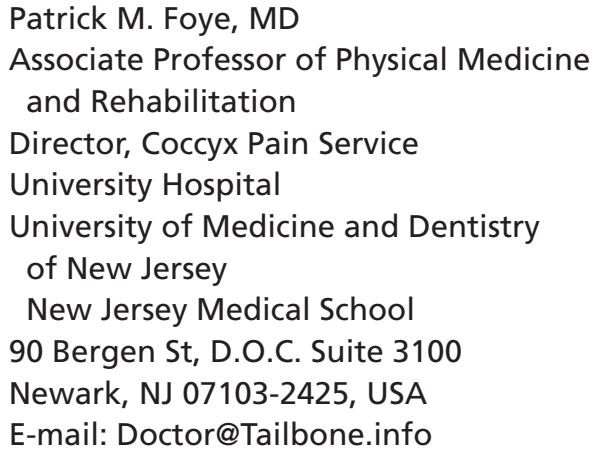

Patrick M. Foye, MD

Associate Professor of Physical Medicine and Rehabilitation

Director, Coccyx Pain Service

University Hospital

University of Medicine and Dentistry of New Jersey New Jersey Medical School 90 Bergen St, D.O.C. Suite 3100 Newark, NJ 07103-2425, USA

E-mail: Doctor@Tailbone.info 


\section{References}

1. Toshniwal GR, Dureja GP, Prashanth SM. Transsacrococcygeal approach to ganglion impar block for management of chronic perineal pain: A prospective observational study. Pain Physician 2007; 10:661-666.

2. Foye PM, Buttaci CJ, Stitik TP, Yonclas PP. Successful injection for coccyx pain. Am J Phys Med Rehabil 2006; 85:783784 .
3. Buttaci CJ, Foye PM, Stitik TP. Coccydynia successfully treated with ganglion impar blocks: A case series. Am J Phys Med Rehabil 2005; 84:218.

4. Foye PM. New approaches to ganglion impar blocks via coccygeal joints. $R e g$ Anesth Pain Med 2007; 32:269.

5. Oh CS, Chung IH, Ji HJ, Yoon DM. Clinical implications of topographic anato- my on the ganglion impar. Anesthesiology 2004; 101:249-250.

6. Postacchini F, Massobrio M. Idiopathic coccygodynia. Analysis of fifty-one operative cases and a radiographic study of the normal coccyx. J Bone Joint Surg Am 1983; 65:1116-1124.

\section{In Response}

We thank Dr. Foye for his interest in our article titled, "Transsacrococcygeal Approach to Ganglion Impar Block for Management of Chronic Perineal Pain: A Prospective Observational Study."(1)

Based on the anatomic study done by Oh et al (2) the new method of injecting Ganglion Impar (GI) through first or second intracoccygeal joints seems attractive (at least, in theory). However, we do not have any personal experience with this technique. The needle through needle technique (3) has been helpful in calcified sacrococcygeal joints most of the time in our experience. Based on the literature available so far, one can only surmise that the position of $\mathrm{Gl}$ is highly variable and therefore any technique will have its own failure rates. The challenge before us is to find a technique that has highest success rate with least side effects. We need to carefully study any new technique rigorously and generate adequate data about its efficacy and the effect on functional outcomes in a multidisciplinary setting. We can then design an adequately powered randomized controlled trial to compare these techniques to find the treatment that best serves our patients.
We again thank Dr. Foye for his interest in our article and also for enlightening us with his experience in this topic.

\section{Gokul R. Toshniwal, MD}

5011, Department of Anesthesia and Intensive Care

All India Institute of Medical Sciences

New Delhi-110021,

India

E-mail: grtosh1@gmail.com

\section{G.P. Dureja, MD}

Department of Pain Medicine

Indian Spinal Injuries Centre, New Delhi.

S.M. Prashanth, MD

Department of Anesthetics

Peterborough and

Stamford Hospitals NHS Trust

United Kingdom.

\section{References}

1. Toshniwal GR, Dureja GP, Prashanth SM. Transsacrococcygeal approach to ganglion impar block for management of chronic perineal pain: A prospective observational study. Pain Physician 2007; 10:661-666.
2. Oh CS, Chung IH, Ji HJ, Yoon DM. Clinical implications of topographic anatomy on the ganglion impar. Anesthesiology 2004; 101:249-250.
Munir MA, Zhang J, Ahmad M. A modified needle in needle technique for the ganglion impar block. Can J Anesth 2004; 51: 915-917. 